\title{
A Polyhedral Off-Line Robust MPC Strategy for Uncertain Polytopic Discrete-Time Systems
}

\author{
Pornchai Bumroongsri and Soorathep Kheawhom* \\ Department of Chemical Engineering, Faculty of Engineering, Chulalongkorn University, Phayathai Rd., \\ Patumwan, Bangkok 10330, Thailand \\ E-mail: Soorathep.k@chula.ac.th*
}

\begin{abstract}
In this paper, an off-line synthesis approach to robust constrained model predictive control for uncertain polytopic discrete-time systems is presented. Most of the computational burdens are moved off-line by pre-computing a sequence of state feedback control laws that corresponds to a sequence of polyhedral invariant sets. The state feedback control laws computed are derived by minimizing the nominal performance cost in order to improve control performance. At each sampling instant, the smallest polyhedral invariant set containing the currently measured state is determined. The corresponding state feedback control law is then implemented to the process. The controller design is illustrated with two examples in chemical processes. The proposed algorithm is compared with an ellipsoidal off-line robust model predictive control algorithm derived by minimizing the worst-case performance cost and an ellipsoidal offline robust model predictive control algorithm derived by minimizing the nominal performance cost. The results show that the proposed algorithm can achieve better control performance. Moreover, a significantly larger stabilizable region is obtained.
\end{abstract}

Keywords: off-line, model predictive control, polyhedral invariant sets, nominal performance cost, chemical processes.

ENGINEERING JOURNAL Volume 16 Issue 4

Received 2 March 2012

Accepted 25 April 2012

Published 1 July 2012

Online at http://www.engj.org/

DOI:10.4186/ej.2012.16.4.73 


\section{Introduction}

Model predictive control (MPC) is an effective control algorithm widely used in chemical process industry. At each sampling time, MPC uses an explicit process model to solve an open-loop optimization problem. Although an optimal control profile is calculated, only the first computed input is implemented to the process. Since model is only an approximation of the real process, one of the main drawbacks of MPC is the difficulty to deal with model uncertainty $[1,2]$. For this reason, synthesis approaches for robust MPC have been widely investigated.

In the work by Kothare et al. [2], robust MPC synthesis which allows an explicit incorporation of model uncertainty in the problem formulation was proposed. The goal is to design the state feedback control law which minimizes the upper bound on the worst-case performance cost. The optimization problem at each time step is formulated as the convex optimization problem involving linear matrix inequalities (LMI). Since the algorithm is derived by using a single Lyapunov function, the algorithm turns out to be very conservative. Moreover, the algorithm requires high on-line computational time because the optimization problem is really solved on-line at each sampling instant.

Robust MPC using multiple Lyapunov functions was proposed in [3]. As compared to the algorithm of Kothare et al. [2], the conservativeness is reduced by using multiple Lyapunov functions instead of a single Lyapunov function. Thus, there are more degrees of freedom in solving the optimization problem. However, the optimization problem needed to be solved on-line at each sampling instant contains many decision variables and constraints.

Another idea to reduce the conservativeness is to increase the degrees of freedom in the optimization problem by using the perturbation of free control inputs $c_{i}, \forall i=1, \ldots, N$. The resulting input sequence is $u_{i}=K x+c_{i}, \forall i=1, \ldots, N-1$ and $u_{i}=K x, \forall i \geq N$. In the work by Brooms et al. [4], robust MPC for uncertain polytopic discrete-time systems with ellipsoidal target sets was presented. The future states are predicted by using a sequence of ellipsoidal sets. The terminal ellipsoidal set is restricted to lie in an ellipsoidal invariant set in order to guarantee robust stability. The perturbation on control input strategy is developed in the controller design in order to ensure feasibility and robust stability. Although robust stability is proved to be guaranteed, the algorithm requires high on-line computational time due to the fact that the number of decision variables and constraints grow significantly with respect to the perturbation horizon $N$.

The perturbation on control input strategy based on parameter-dependent Lyapunov function was presented in [5]. The control law derived by parameter-dependent Lyapunov function is perturbed by a sequence of free control inputs over the perturbation horizon $N$ in order to improve the control performance. At each sampling time $k$, the terminal invariant set containing the terminal state is constructed in order to guarantee robust stability. The algorithm can reduce the conservativeness because both the feedback gain and the free control inputs are derived from parameter-dependent Lyapunov function. However, the effect of plant uncertainty is still overestimated because both the control law and the free control inputs are derived by minimizing the worst-case performance cost. Moreover, the computational time grows significantly with the perturbation horizon $N$.

The on-line robust MPC usually requires high computational time. Thus, its ability is limited to the relatively slow dynamic processes. In order to reduce on-line computational demand, a number of researchers have studied off-line robust MPC. In the work by Wan and Kothare [6], an off-line formulation of robust MPC using linear matrix inequalities was presented. A sequence of control laws corresponding to a sequence of invariant ellipsoids is computed off-line. At each sampling time, the smallest ellipsoid containing the measured state is determined and the corresponding control law is implemented to the process. Although the algorithm substantially reduces on-line computational time, the conservative result is obtained. This is due to the fact that the invariant ellipsoids constructed are only the approximations of the true polyhedral invariant sets. An ellipsoidal off-line robust MPC algorithm based on nominal performance cost was presented by Ding et al. [7]. The algorithm directly extends the algorithm of Wan and Kothare [6] by choosing the nominal performance cost to substitute the worst-case performance cost in order to improve control performance.

In [8], an ellipsoidal off-line MPC scheme for uncertain polytopic discrete-time systems was developed. The ellipsoidal inner approximations of the exact controllable sets are computed off-line and a numerically low demanding optimization problem is solved on-line. Although most of the numerical burdens are moved off-line, the feasible region is significantly smaller than the polyhedral counterpart. This is due to the fact that the controllable ellipsoidal sets constructed are only the approximations of the true controllable 
polyhedral sets. Moreover, the algorithm requires a large number of controllable ellipsoidal sets in order to ensure feasibility.

In [9], an ellipsoidal off-line MPC algorithm for LPV systems was proposed. The sequences of state feedback gains corresponding to the sequences of nested ellipsoids are computed off-line. At each sampling instant, the smallest ellipsoid containing the currently measured state is determined in each sequence of ellipsoids and the scheduling parameter is measured. The real-time state feedback gain is then calculated by linear interpolation between the corresponding state feedback gains.

From the preceding review, we can see that the on-line robust MPC algorithms usually require high computational time. Thus, they are computationally prohibitive in practical situations. For the off-line robust MPC algorithms, the ellipsoidal approximations of the exact polyhedral invariant sets are usually used. Thus, the algorithms turn out to be very conservative. Moreover, the stabilizable region is apparently smaller than the polyhedral counterpart. In this paper, we present an off-line synthesis approach to robust MPC using polyhedral invariant sets. A sequence of state feedback control laws that corresponds to a sequence of polyhedral invariant sets is computed off-line. Then the smallest polyhedral invariant set containing the currently measured state is determined on-line and the corresponding state feedback control law is implemented to the process.

The paper is organized as follows. In section 2 , the problem description is presented. In section 3 , the polyhedral off-line robust MPC algorithm is presented. In section 4, we present two examples in chemical processes to illustrate our algorithm. Finally, in section 5, we conclude the paper.

Notation: For a matrix $A, A^{T}$ denotes its transpose, $A^{-1}$ denotes its inverse. $I$ denotes the identity matrix. For a vector $x, x(k / k)$ denotes the state measured at real time $k, x(k+i / k)$ denotes the state at prediction time $k+i$ predicted at real time $k \cdot \hat{x}(k+i / k)$ denotes the nominal state at prediction time $k+i$ predicted at real time $k \cdot[\hat{A}, \hat{B}]$ denotes the model that is more likely to be the actual plant. The symbol * denotes the corresponding transpose of the lower block part of symmetric matrices. The matrix inequality $A>B(A \geq B)$ means that $A$ and $B$ are square symmetric and $A-B$ is positive (semi-) definite. The Lyapunov function $V(i, k)$ is defined as $V(i, k)=x(k+i / k)^{T} P(i, k) x(k+i / k)$ where $\forall k, \forall i \geq 0, P(i, k)>0$.

\section{Problem Description}

The model considered here is the following linear time varying (LTV) system with polytopic uncertainty

$$
\begin{aligned}
& x(k+1)=A(k) x(k)+B(k) u(k) \\
& y(k)=C x(k)
\end{aligned}
$$

where $x(k)$ is the state of the plant, $u(k)$ is the control input and $y(k)$ is the plant output. Moreover, we assume that

$$
[A(k), B(k)] \in \Omega, \Omega=\operatorname{Co}\left\{\left[A_{1}, B_{1}\right],\left[A_{2}, B_{2}\right], \ldots,\left[A_{L}, B_{L}\right]\right\}
$$

where $\Omega$ is the polytope, $\boldsymbol{C o}$ denotes convex hull, $\left[A_{j}, B_{j}\right]$ are vertices of the convex hull. Any $[A(k), B(k)]$ within the polytope $\Omega$ is a linear combination of the vertices such that

$$
[A(k), B(k)]=\sum_{j=1}^{L} \lambda_{j}\left[A_{j}, B_{j}\right], \sum_{j=1}^{L} \lambda_{j}=1,0 \leq \lambda_{j} \leq 1
$$

where $\lambda=\left[\lambda_{1}, \lambda_{2}, \ldots, \lambda_{L}\right]$ is the uncertain parameter vector. The aim of this research is to find the state feedback control law

$$
u(k+i / k)=K x(k+i / k)
$$


which stabilizes the system (2.1) and achieves the following nominal performance cost

$$
\begin{gathered}
\min _{u(k+i / k), i \geq 0} \max J_{n, \infty}(k) \\
J_{n, \infty}(k)=\sum_{i=0}^{\infty}\left[\begin{array}{c}
\hat{x}(k+i / k) \\
u(k+i / k)
\end{array}\right]^{T}\left[\begin{array}{cc}
\Theta & 0 \\
0 & R
\end{array}\right]\left[\begin{array}{c}
\hat{x}(k+i / k) \\
u(k+i / k)
\end{array}\right]
\end{gathered}
$$

where $\Theta>0$ and $R>0$ are symmetric weighting matrices subject to input and output constraints

$$
\begin{gathered}
\left|u_{h}(k+i / k)\right| \leq u_{h, \max }, h=1,2,3, \ldots, n_{u} \\
\left|y_{r}(k+i / k)\right| \leq y_{r, \max }, r=1,2,3, \ldots, n_{y}
\end{gathered}
$$

\section{The Polyhedral Off-Line Robust MPC Algorithm}

An off-line formulation of robust MPC using linear matrix inequalities (LMI) was first developed by Wan and Kothare [6]. The on-line computational time is reduced by computing off-line a sequence of control laws corresponding to a sequence of ellipsoidal invariant sets. At each sampling time, the smallest ellipsoid containing the measured state is determined and the corresponding control law is implemented to the process. Although the algorithm substantially reduces on-line computational time, the conservative result is obtained due to the fact that the invariant ellipsoids constructed are only the approximations of the true polyhedral invariant sets. Moreover, the state feedback gain is derived by minimizing the worst-case performance cost. Thus, the effect of model uncertainty is overestimated.

In this section, an off-line synthesis approach to robust MPC using polyhedral invariant sets is presented. The on-line computational time is reduced by pre-computing off-line a sequence of state feedback control laws corresponding to a sequence of polyhedral invariant sets. The state feedback gains computed are derived by minimizing the nominal performance cost [7] in order to improve control performance. The approach to construct the polyhedral invariant set developed by Pluymers et al. [10] is adopted here to construct a sequence of polyhedral invariant sets. At each sampling time, the smallest polyhedral invariant set containing the currently measured state is determined and the corresponding state feedback control law is then implemented to the process.

The definition of polyhedral invariant set is given as follows:

Definition 3.1: The set $S=\{x / M x \leq d\}$ is said to be the polyhedral invariant set if it has the property that whenever $x(k) \in S$, then $x(k+i) \in S, \forall i=1,2, \ldots, \infty$.

We can now formulate an off-line robust MPC algorithm using polyhedral invariant sets. Our algorithm consists of two steps. In the first step, a sequence of state feedback gains corresponding to a sequence of ellipsoidal invariant sets is calculated by minimizing the nominal performance cost. A sequence of polyhedral invariant sets is then constructed in the second step. A sequence of ellipsoidal invariant sets obtained in the first step is identical to that of Ding et al. [7]. Instead of directly using a sequence of ellipsoidal invariant sets, in our algorithm, we further improved the control performance of the off-line MPC by generating a sequence of polyhedral invariant sets in the second step.

\section{Algorithm 3.1:}

\section{Off-line:}

Step 1: Choose a sequence of states $x_{i}, i \in\{1,2, \ldots, N\}$. For each $x_{i}$, solve the following problem to obtain the corresponding state feedback gain $K_{i}=Y_{i} Q_{i}^{-1}$ where $Y_{i}$ and $Q_{i}$ are the matrix variables, $\gamma_{n, i}$ is the 
upper bound on the nominal performance cost. The states $x_{i}$ should be chosen such that the distance between $x_{i+1}$ and the origin is less than the distance between $x_{i}$ and the origin.

$$
\begin{aligned}
& \min _{Y_{i}, Q_{i}} \gamma_{n, i} \\
\text { s.t. } & {\left[\begin{array}{cc}
1 & * \\
x_{i} & Q_{i}
\end{array}\right] \geq 0 } \\
& {\left[\begin{array}{cccc}
Q_{i} & * & * & * \\
A Q_{i}+B Y_{i} & Q_{i} & * & * \\
\Theta^{\frac{1}{2}} Q_{i} & 0 & \gamma_{n, i} I & * \\
R^{\frac{1}{2}} Y_{i} & 0 & 0 & \gamma_{n, i} I
\end{array}\right] \geq 0 } \\
& {\left[\begin{array}{cc}
Q_{i} & * \\
A_{j} Q_{i}+B_{j} Y_{i} & Q_{i}
\end{array}\right]>0, \forall j=1,2, \ldots, L } \\
& {\left[\begin{array}{cr}
X & * \\
Y_{i}^{T} & Q_{i}
\end{array}\right] \geq 0, X_{h h} \leq u_{h, \max }^{2}, h=1,2, \ldots, n_{u} } \\
& {\left[\begin{array}{rr}
S \\
\left(A_{j} Q_{i}+B_{j} Y_{i}\right)^{T} C^{T} & Q_{i}
\end{array}\right] \geq 0, \forall j=1,2, \ldots, L, S_{r r} \leq y_{r, \max }^{2}, r=1,2, \ldots, n_{y} }
\end{aligned}
$$

Step 2: Given a sequence of state feedback gains $K_{i}=Y_{i} Q_{i}^{-1}, i=1,2, \ldots, N$ from step 1 . For each $K_{i}$, the corresponding polyhedral invariant set $S_{i}=\left\{x / M_{i} x \leq d_{i}\right\}$ is constructed by following these steps:

2.1) Set $M_{i}=\left[C^{T},-C^{T}, K_{i}^{T},-K_{i}^{T}\right]^{T}, d_{i}=\left[y_{\max }^{T}, y_{\min }^{T}, u_{\max }^{T}, u_{\min }^{T}\right]^{T}$ and $m=1$

2.2) Select row $m$ from $\left(M_{i}, d_{i}\right)$ and check $\forall j$ whether $M_{i, m}\left(A_{j}+B_{j} K_{i}\right) x \leq d_{i, m}$ is redundant with respect to the constraints defined by $\left(M_{i}, d_{i}\right)$ by solving the following problem:

$$
\begin{aligned}
& \max _{x} W_{i, m, j} \\
& \text { s.t. } W_{i, m, j}=M_{i, m}\left(A_{j}+B_{j} K_{i}\right) x-d_{i, m} \\
& M_{i} x \leq d_{i}
\end{aligned}
$$

If $W_{i, m, j}>0$, the constraint $M_{i, m}\left(A_{j}+B_{j} K_{i}\right) x \leq d_{i, m}$ is non-redundant with respect to $\left(M_{i}, d_{i}\right)$. Then, add non-redundant constraints to $\left(M_{i}, d_{i}\right)$ by assigning

$$
M_{i}=\left[M_{i}^{T},\left(M_{i, m}\left(A_{j}+B_{j} K_{i}\right)\right)^{T}\right]^{T} \text { and } d_{i}=\left[d_{i}^{T}, d_{i, m}^{T}\right]^{T}
$$

2.3) Let $m=m+1$ and return to step 2.2. If $m$ is strictly larger than the number of rows in $\left(M_{i}, d_{i}\right)$ then terminate. 
On-line: At each sampling time, determine the smallest polyhedral invariant set $S_{i}=\left\{x / M_{i} x \leq d_{i}\right\}$ containing the measured state and implement the corresponding state feedback control law $u(k / k)=K_{i} x(k / k)$ to the process.

Remark: In an off-line step 1, a sequence of feedback gains corresponding to a sequence of ellipsoidal invariant sets is calculated. Although each feedback gain calculated guarantees robust stability within the corresponding ellipsoidal invariant set, this ellipsoidal invariant set is only an approximation of the true polyhedral invariant set. By using only an off-line step 1, the conservative result is obtained because the stabilizable region of the ellipsoidal invariant set is significantly smaller than the polyhedral counterpart. This problem is especially severe in the case of tight constraints. Thus, for a given sequence of feedback gains calculated from an off-line step 1, a sequence of true polyhedral invariant sets is calculated in an offline step 2. By using an off-line step 2, the conservativeness is reduced because the stabilizable region of each feedback gain is substantially expanded.

An overall algorithm is proved to guarantee robust stability in Theorem 3.1.

Theorem 3.1 Given the initial measured state $x(k) \in S_{1}$, the control law provided by algorithm 3.1 assures robust stability to the closed-loop system.

Proof. The satisfaction of (3.3) for the state feedback gain $K_{i}=Y_{i} Q_{i}^{-1}$ ensures that

$$
x(k+i / k)^{T}\left\{\left[A(k+i)+B(k+i) K_{i}\right]^{T} P(i+1, k)\left[A(k+i)+B(k+i) K_{i}\right]-P(i, k)\right\} x(k+i / k)<0
$$

Thus, $V(i, k)=x(k+i / k)^{T} P(i, k) x(k+i / k)$ is a strictly decreasing Lyapunov function and the closedloop system is robustly stabilized by the state feedback gain $K_{i}$.

By solving (3.6) and iteratively adding non-redundant constraints $M_{i, m}\left(A_{j}+B_{j} K_{i}\right) x \leq d_{i, m}$ to $\left(M_{i}, d_{i}\right)$ by assigning $M_{i}=\left[M_{i}^{T},\left(M_{i, m}\left(A_{j}+B_{j} K_{i}\right)\right)^{T}\right]^{T}$ and $d_{i}=\left[d_{i}^{T}, d_{i, m}^{T}\right]^{T}$, we can find the set of initial states $x$ defined by $S_{i}=\left\{x / M_{i} x \leq d_{i}\right\}$ such that all future states are guaranteed to stay within this set without input and output constraints violation. Any initial states outside $S_{i}$ lead to the future states that violate input and output constraints for at least one realization of the uncertainty.

Thus, the set $S_{i}$ is polyhedral invariant set and the corresponding state feedback control law $u(k+i / k)=K_{i} x(k+i / k)$ assures robust stability to the closed-loop system.

\section{Examples}

In this section, we present two examples that illustrate the implementation of the proposed MPC algorithm. For both examples, the numerical simulations have been performed in Intel Core i-5 (2.4 GHz), $2 \mathrm{~GB}$ RAM, using SeDuMi [11] and YALMIP [12] within Matlab R2008a environment.

\section{Example 4.1:}

In the first example, we will consider the application of our approach to an uncertain non-isothermal CSTR where the exothermic reaction $A \longrightarrow B$ takes place. The reaction is irreversible and the rate of reaction is first order with respect to component $A$. A cooling coil is used to remove heat that is released in the exothermic reaction. The reaction rate constant $k_{o}$ and the heat of reaction $\Delta H_{r x n}$ are considered to be the uncertain parameters. They are assumed to be arbitrarily time-varying in the indicated range of variation. The linearized model based on the component balance and the energy balance is given as follows [6]: 


$$
\begin{aligned}
& {\left[\begin{array}{c}
\dot{C_{A}} \\
\dot{T}
\end{array}\right]=\left[\begin{array}{cc}
-\frac{F}{V}-k_{o} e^{-\frac{E}{R T_{S}}} & -\frac{E}{R T_{S}^{2}} k_{o} e^{-\frac{E}{R T_{S}}} C_{A S} \\
\frac{-\Delta H_{r x n} k_{o} e^{-\frac{E}{R T_{S}}}}{\rho C_{p}} & -\frac{F}{V}-\frac{U A}{V \rho C_{p}}-\Delta H_{r x n} \frac{E}{\rho C_{p} R T_{S}^{2}} k_{o} e^{-\frac{E}{R T_{S}}} C_{A S}
\end{array}\right]\left[\begin{array}{c}
C_{A} \\
T
\end{array}\right]+\left[\begin{array}{cc}
\frac{F}{V} & 0 \\
0 & -2.098 x 10^{5} \frac{T_{S}-365}{V \rho C_{p}}
\end{array}\right]\left[\begin{array}{c}
C_{A, F} \\
F_{C}
\end{array}\right]} \\
& y=\left[\begin{array}{ll}
1 & 0 \\
0 & 1
\end{array}\right]\left[\begin{array}{c}
C_{A} \\
T
\end{array}\right]
\end{aligned}
$$

where $C_{A}$ is the concentration of $A$ in the reactor, $C_{A, F}$ is the feed concentration of $A, T$ is the reactor temperature and $F_{c}$ is the coolant flow. The operating parameters are shown in Table 4.1.

Table 4.1. The operating parameters of non-isothermal CSTR in Example 4.1.

\begin{tabular}{lcr}
\hline Parameter & Value & Unit \\
\hline$F$ & 1 & $\mathrm{~m}^{3} / \mathrm{min}^{3}$ \\
$V$ & 1 & $\mathrm{~m}^{3}$ \\
$\rho$ & $10^{6}$ & $\mathrm{~g} / \mathrm{m}^{3}$ \\
$C_{p}$ & 1 & $\mathrm{cal} / \mathrm{g} \cdot \mathrm{K}$ \\
$-\Delta H_{r x n}$ & $10^{7}-10^{8}$ & $\mathrm{cal} / \mathrm{kmol}$ \\
$E / R$ & 8330.1 & $\mathrm{~K}$ \\
$k_{o}$ & $10^{9}-10^{10}$ & $\mathrm{~min}^{-1}$ \\
$U A$ & $5.34 \times 10^{6}$ & $\mathrm{cal} / \mathrm{K} \cdot \mathrm{min}^{2}$ \\
\hline
\end{tabular}

Let $\overline{C_{A}}=C_{A}-C_{A, e q}, \bar{T}=T-T_{e q}, \bar{C}_{A, F}=C_{A, F}-C_{A, F, e q}$ and $\bar{F}_{c}=F_{c}-F_{c, e q}$, where the subscript $e q$ is used to denote the corresponding variable at equilibrium condition. The discrete-time model (4.2) is obtained by discretizing (4.1) using Euler first-order approximation with a sampling time of $0.15 \mathrm{~min}$.

$$
\begin{gathered}
{\left[\begin{array}{c}
\bar{C}_{A}(k+1) \\
\bar{T}(k+1)
\end{array}\right]=\left[\begin{array}{cc}
0.85-0.0986 \alpha(k) & -0.0014 \alpha(k) \\
0.9864 \alpha(k) \beta(k) & 0.0487+0.01403 \alpha(k) \beta(k)
\end{array}\right]\left[\begin{array}{c}
\bar{C}_{A}(k) \\
\bar{T}(k)
\end{array}\right]+\left[\begin{array}{cc}
0.15 & 0 \\
0 & -0.912
\end{array}\right]\left[\begin{array}{c}
\bar{C}_{A, F}(k) \\
\bar{F}_{C}(k)
\end{array}\right]} \\
\bar{y}(k)=\left[\begin{array}{cc}
1 & 0 \\
0 & 1
\end{array}\right]\left[\begin{array}{c}
\bar{C}_{A}(k) \\
\bar{T}(k)
\end{array}\right]
\end{gathered}
$$

where $1 \leq \alpha(k)=k_{o} / 10^{9} \leq 10$ and $1 \leq \beta(k)=-\Delta H_{r x n} / 10^{7} \leq 10$. Because two uncertain parameters $\alpha(k)$ and $\beta(k)$ are independent of each other, we have to consider the polytopic uncertain model with its four vertices representing all the possible combinations of the two uncertain parameters. The polytopic uncertain set is given as follows

$$
\Omega=\operatorname{Co}\left\{\left[\begin{array}{cc}
0.751 & -0.0014 \\
0.986 & 0.063
\end{array}\right],\left[\begin{array}{cc}
0.751 & -0.0014 \\
9.864 & 0.189
\end{array}\right],\left[\begin{array}{cc}
-0.136 & -0.014 \\
9.864 & 0.189
\end{array}\right],\left[\begin{array}{cc}
-0.136 & -0.014 \\
98.644 & 1.451
\end{array}\right]\right\}
$$


The objective is to regulate $\bar{C}_{A}$ and $\bar{T}$ by manipulating $\bar{C}_{A, F}$ and $\bar{F}_{c}$, respectively. The input constraints are $\left|\bar{C}_{A, F}\right| \leq 0.5 \mathrm{kmol} / \mathrm{m}^{3}$ and $\left|\overline{F_{C}}\right| \leq 1.5 \mathrm{~m}^{3} / \mathrm{min}$. Here $J_{n, \infty}(k)$ is given by (2.5) with $\Theta=I$ and $R=0.1 I$. The nominal model is given by $\hat{A}=\left[\begin{array}{ll}0.357 & -0.007 \\ 24.66 & 0.3990\end{array}\right]$ and $\hat{B}=\left[\begin{array}{cc}0.15 & 0 \\ 0 & -0.912\end{array}\right]$.

Figure 4.1 shows the comparison among a) the polyhedral invariant sets constructed off-line by algorithm 3.1, b) the ellipsoidal invariant sets constructed off-line based on the worst-case performance cost as proposed by Wan and Kothare [6] and c) the ellipsoidal invariant sets constructed off-line based on the nominal performance cost as proposed by Ding et al. [7]. Note that for all algorithms, the invariant sets are constructed by choosing the same sequence of states $x_{i}=\{(0.0525,0.0525),(0.0475,0.0475),(0.0425,0.0425),(0.0375,0.0375),(0.0325,0.0325),(0.0275,0.0275)\}$.

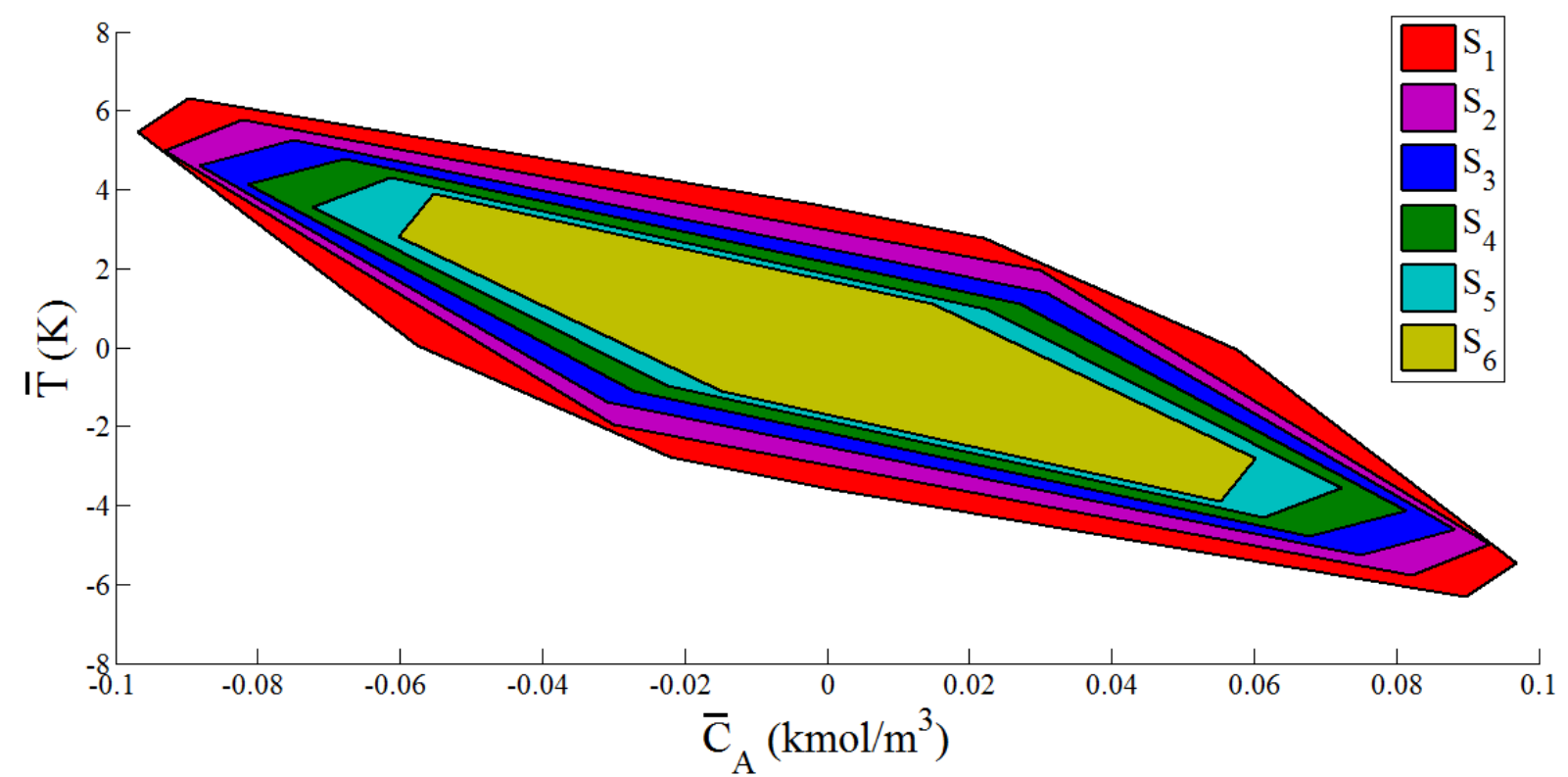

(a) The polyhedral invariant sets $S_{i}, i=1, \ldots, 6$ constructed off-line by algorithm 3.1. 


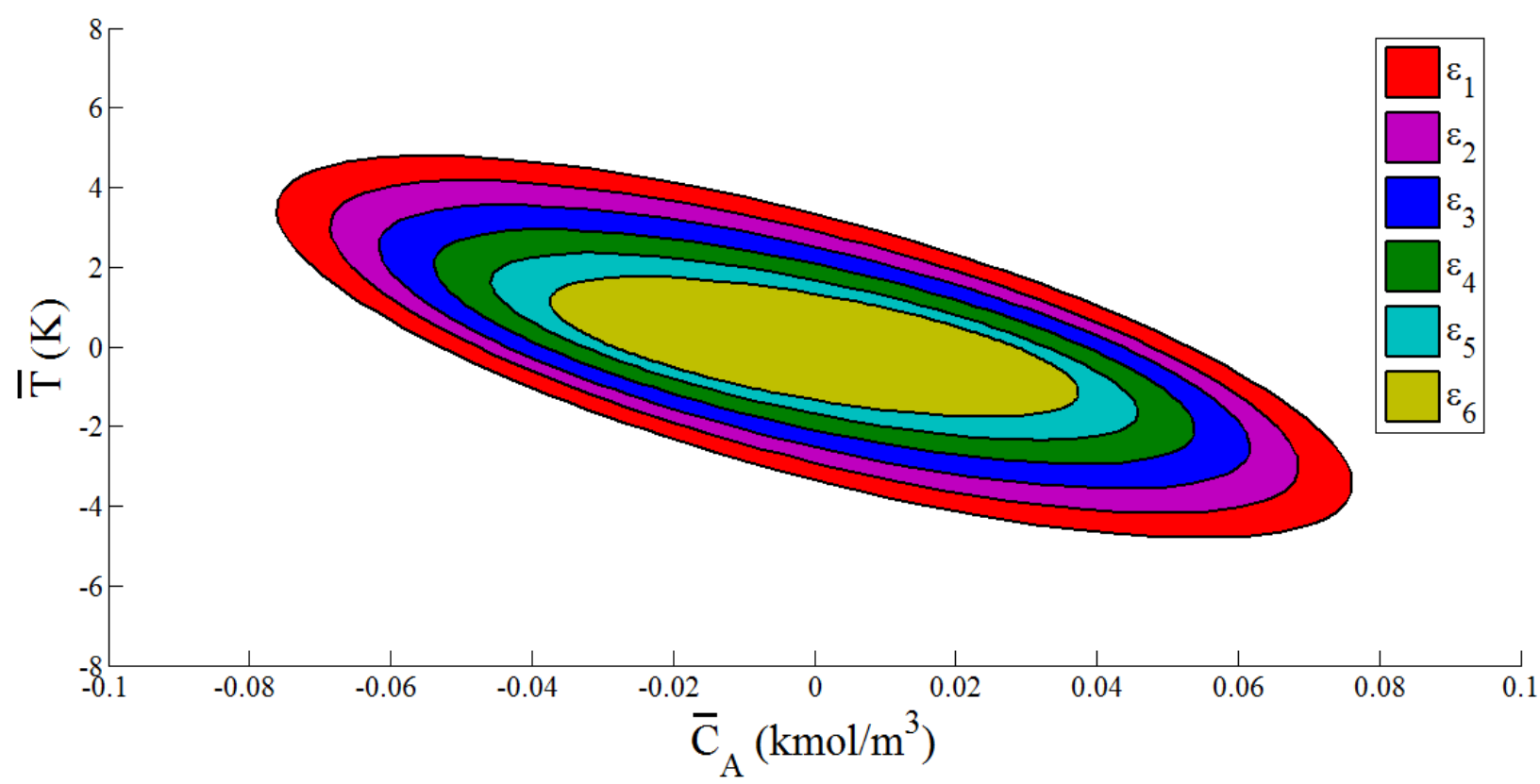

(b) The ellipsoidal invariant sets $\varepsilon_{i}, i=1, \ldots, 6$ constructed off-line based on the worst-case performance cost .

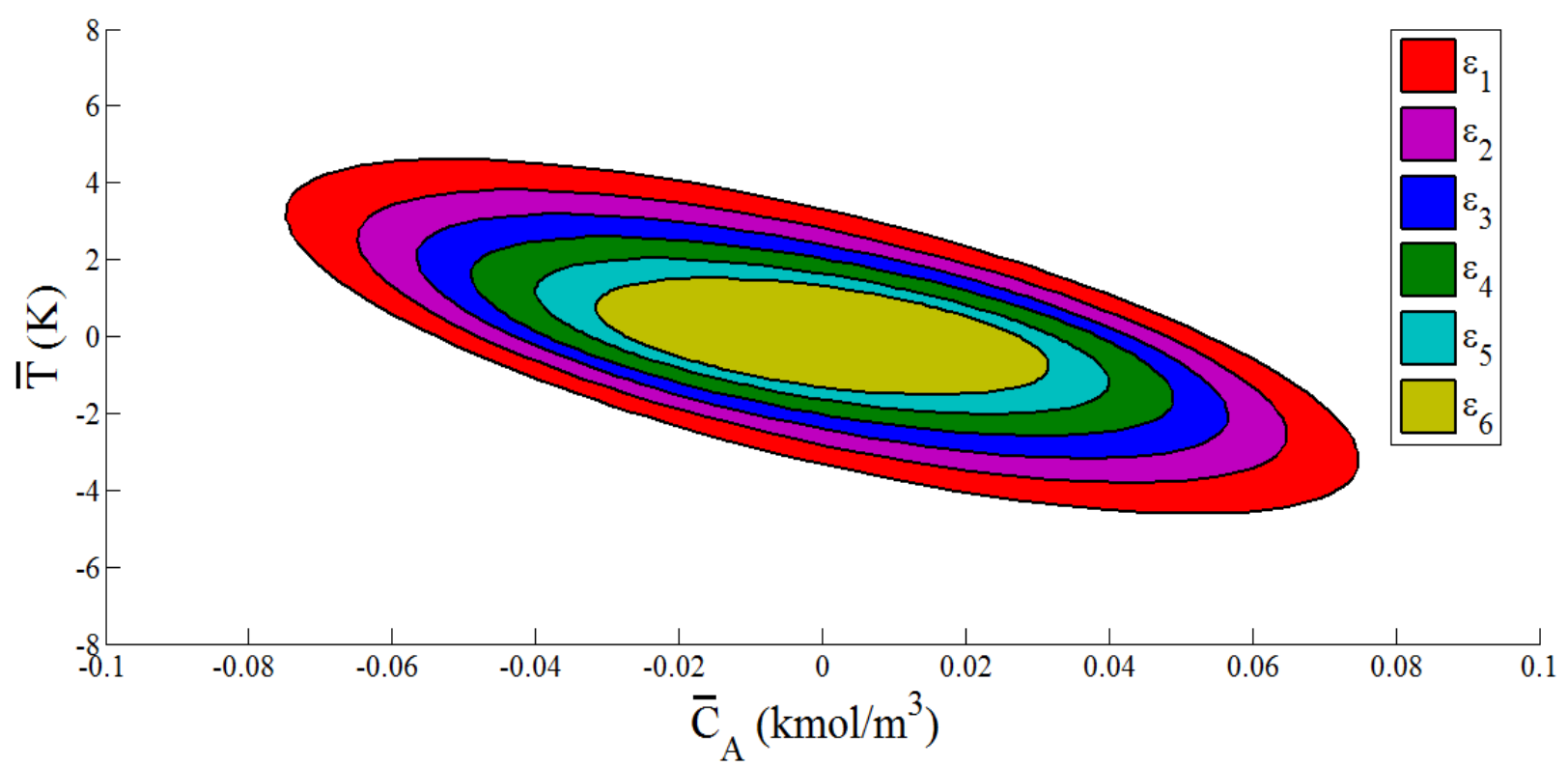

(c) The ellipsoidal invariant sets $\varepsilon_{i}, i=1, \ldots, 6$ constructed off-line based on the nominal performance cost .

Fig. 4.1. The comparison among (a) The polyhedral invariant sets constructed off-line by algorithm 3.1, (b) the ellipsoidal invariant sets constructed off-line based on the worst-case performance cost and (c) the ellipsoidal invariant sets constructed off-line based on the nominal performance cost. For all algorithms, the invariant sets are constructed by choosing the same sequence of states $x_{i}=\{(0.0525,0.0525),(0.0475,0.0475),(0.0425,0.0425),(0.0375,0.0375),(0.0325,0.0325)$,

$(0.0275,0.0275)\}$.

Figure 4.2 shows the stabilizable regions of feedback gains $K_{1}$ and $K_{2}$. In this example, $K_{2}$ is larger than $K_{1}$ because $K_{2}$ is computed by using the state which is closer to the origin than $K_{1}$. Note that each feedback gain $K_{\mathrm{i}}$ of algorithm 3.1 and $K_{\mathrm{i}}$ of an ellipsoidal off-line robust MPC algorithm based on the nominal performance cost are identical because they are all derived by minimizing the nominal performance cost. In comparison, each $K_{\mathrm{i}}$ of an ellipsoidal off-line robust MPC algorithm based on the worst-case performance cost is derived by minimizing the worst-case performance cost and hence it can entail a certain degree of 
conservativeness because the effect of plant uncertainty is overestimated. It can be observed from the figure that an ellipsoidal off-line robust MPC algorithm based on the worst-case performance cost and an ellipsoidal off-line robust MPC algorithm based on the nominal performance cost cannot stabilize the state at point $\mathrm{A}$. This is due to the fact that the state at point $\mathrm{A}$ is not contained in the largest invariant ellipsoid $x_{\text {point A }} \notin \varepsilon_{1}$. In comparison, algorithm 3.1 can stabilize the state at point A because the state is contained in the largest polyhedral invariant set $x_{\text {point A }} \in S_{1}$. It can also be observed from the figure that if we start at point B, an ellipsoidal off-line robust MPC algorithm based on the worst-case performance cost and an ellipsoidal off-line robust MPC algorithm based on the nominal performance cost can stabilize the state by using the lowest feedback gain $K_{1}$ because $x_{\text {point В }} \in \varepsilon_{1}$. In comparison, the algorithm 3.1 can stabilize the state by using higher feedback gain $K_{2}$ due to the fact that $x_{\text {point B }} \in S_{2}$. In this circumstance, algorithm 3.1 can adopt higher feedback gain as compared with an ellipsoidal off-line robust MPC algorithm based on the worst-case performance cost and an ellipsoidal off-line robust MPC algorithm based on the nominal performance cost. Thus, algorithm 3.1 can achieve less conservative result.

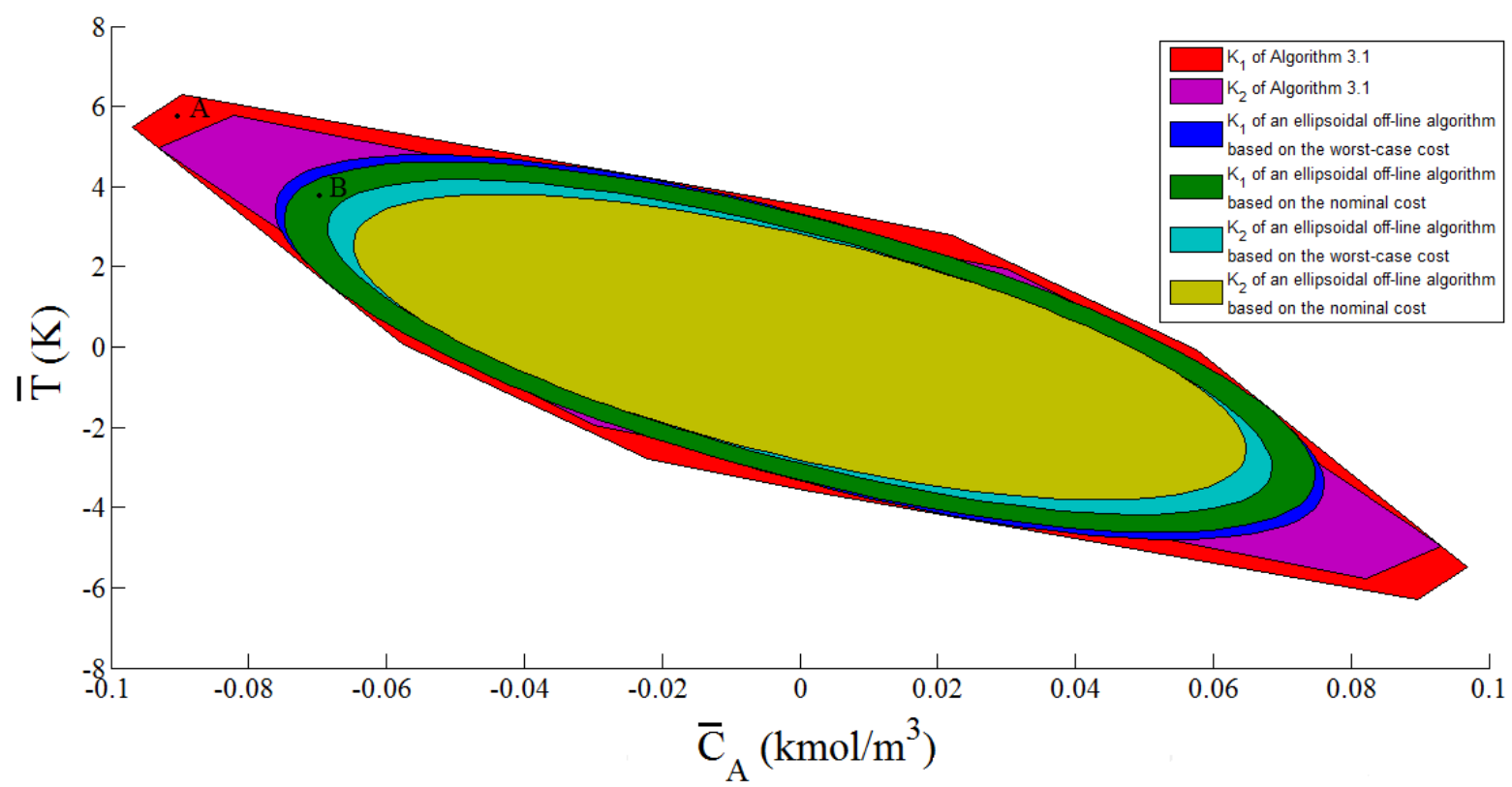

Fig. 4.2. The stabilizable regions of feedback gains $K_{1}$ and $K_{2}$.

Figure 4.3 shows the closed-loop responses of the system when $\alpha(k)$ and $\beta(k)$ are randomly timevarying between $10^{9} \leq \alpha(k)=k_{o} \leq 10^{10}$ and $10^{7} \leq \beta(k)=-\Delta H_{r x n} \leq 10^{8}$. It can be observed from the figure that algorithm 3.1 can achieve less conservative result as compared with an ellipsoidal off-line robust MPC algorithm based on the worst-case performance cost and an ellipsoidal off-line robust MPC algorithm based on the nominal performance cost. 

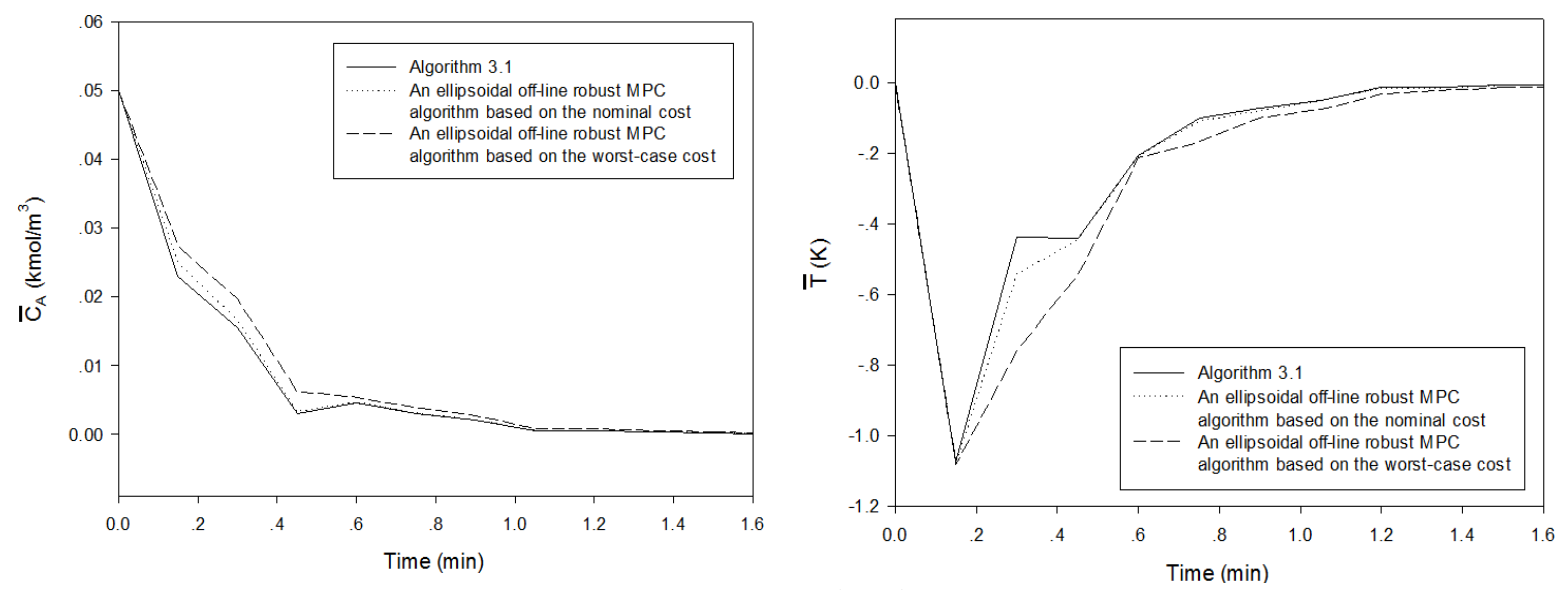

(a) Regulated output
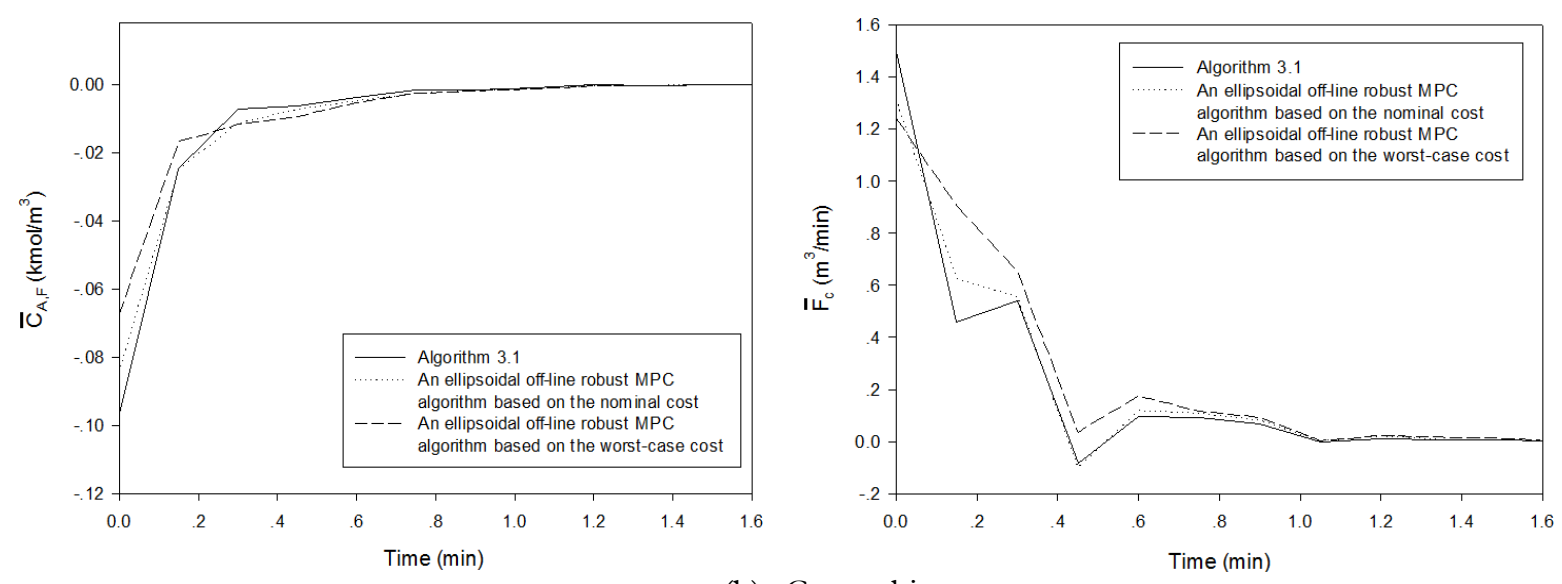

(b) Control input

Fig. 4.3. The closed-loop responses of the system when $\alpha(k)$ and $\beta(k)$ are randomly time-varying between $10^{9} \leq \alpha(k)=k_{o} \leq 10^{10}$ and $10^{7} \leq \beta(k)=-\Delta H_{r x n} \leq 10^{8}$.

For an ellipsoidal off-line robust MPC algorithm based on the worst-case performance cost, the on-line computational time is reduced by computing off-line a sequence of control laws corresponding to a sequence of ellipsoidal invariant sets. Although the algorithm substantially reduces on-line computational time, the conservative result is obtained. This is due to the fact that the invariant ellipsoids constructed are only the approximations of the true polyhedral invariant sets. Moreover, the state feedback gain is derived by minimizing the worst-case performance cost that is not likely to occur. Thus, the effect of model uncertainty is overestimated. For the algorithm 3.1, most of the computational burdens are moved off-line by pre-computing a sequence of state feedback control laws corresponding to a sequence of polyhedral invariant sets. The state feedback gains computed are derived by minimizing the nominal performance cost. Thus, the algorithm 3.1 can achieve better control performance as compared with an ellipsoidal off-line robust MPC algorithm based on the worst-case performance cost because the effect of model uncertainty is estimated based on the most likely or nominal scenario. Moreover, the algorithm 3.1 is derived based on the polyhedral invariant sets. Thus, it can obtain a significantly larger stabilizable region as compared with an ellipsoidal off-line robust MPC algorithm based on the worst-case performance cost.

\section{Example 4.2:}

In the second example, we will consider the application of our approach to a continuous bioreactor in fermentation process. Biochemical reactors are used to produce a large number of products including pharmaceuticals, food and beverages. In this bioreactor model, only two components are considered 
including biomass $X_{B}$ and substrate $S$. A fermentation process is assumed to occur in an isothermal continuous bioreactor with constant volume and constant physical-chemical properties. The maximum growth rate $\mu_{\max }$ of biomass is considered to be the uncertain parameter. It is assumed to be arbitrarily time-varying in the indicated range of variation. The dynamic model based on the component balance is given as follows [13]:

$$
\begin{aligned}
& \frac{d X_{B}}{d t}=\mu(S) X_{B}-X_{B} D \\
& \frac{d S}{d t}=-\frac{\mu(S) X_{B}}{Y}+\left(S_{F}-S\right) D \\
& \mu(S)=\mu_{\max } \frac{S}{K_{2} S^{2}+S+K_{1}} \\
& \mathrm{y}=\left[\begin{array}{ll}
1 & 0 \\
0 & 1
\end{array}\right]\left[\begin{array}{c}
X_{B} \\
S
\end{array}\right]
\end{aligned}
$$

where $X_{B}$ is the biomass concentration, $S$ is the substrate concentration and $D$ is the dilution rate. The operating parameters are shown in Table 4.2.

Table 4.2. The operating parameters of continuous bioreactor in example 4.2.

\begin{tabular}{lcr}
\hline Parameter & Value & Unit \\
\hline$S_{F}$ & 4 & $\mathrm{~kg} / \mathrm{m}^{3}$ \\
$Y$ & 0.4 & - \\
$\mu_{\max }$ & $0.01-0.99$ & $\mathrm{hr}^{-1}$ \\
$K_{1}$ & 0.12 & $\mathrm{~kg} / \mathrm{m}^{3}$ \\
$K_{2}$ & 0.4545 & $\mathrm{~m}^{3} / \mathrm{kg}$ \\
\hline
\end{tabular}

Let $\overline{X_{B}}=X_{B}-X_{B, e q}, \bar{S}=S-S_{e q}$ and $\bar{D}=D-D_{e q}$. The discrete-time model (4.5) is obtained by linearization and discretization of (4.4) using Euler first-order approximation with a sampling time of $0.2 \mathrm{hr}$.

$$
\begin{gathered}
{\left[\begin{array}{l}
\overline{X_{B}}(k+1) \\
\bar{S}(k+1)
\end{array}\right]=\left[\begin{array}{cc}
0.1132 \mu_{\max }(k)+0.94 & 0.3414 \mu_{\max }(k) \\
-0.283 \mu_{\max }(k) & -0.8536 \mu_{\max }(k)+0.94
\end{array}\right]\left[\begin{array}{c}
\overline{X_{B}}(k) \\
\bar{S}(k)
\end{array}\right]+\left[\begin{array}{c}
-0.3060 \\
0.7651
\end{array}\right] \bar{D}(k)} \\
\bar{y}(k)=\left[\begin{array}{cc}
1 & 0 \\
0 & 1
\end{array}\right]\left[\begin{array}{c}
\overline{X_{B}}(k) \\
\bar{S}(k)
\end{array}\right]
\end{gathered}
$$

where $0.01 \leq \mu_{\max }(k) \leq 0.99$. Because the uncertain parameter $\mu_{\max }(k)$ is varied between 0.01 and 0.99 , we conclude that $A(k) \in \Omega$ where $\Omega$ is given as follows

$$
\Omega=\operatorname{Co}\left\{\left[\begin{array}{cc}
0.9411 & 0.0034 \\
-0.0028 & 0.9314
\end{array}\right],\left[\begin{array}{cc}
1.0521 & 0.3380 \\
-0.2802 & 0.0949
\end{array}\right]\right\}
$$


The objective is to regulate $\overline{X_{B}}$ and $\bar{S}$ by manipulating $\bar{D}$. The input constraint is $|\bar{D}(k)| \leq 0.015 \mathrm{hr}^{-1}$. Here $J_{n, \infty}(k)$ is given by (2.5) with $\Theta=I$ and $R=0.1 I$. The nominal model is given by $\hat{A}=\left[\begin{array}{cc}0.9994 & 0.1809 \\ -0.1500 & 0.4876\end{array}\right]$ and $\hat{B}=\left[\begin{array}{c}-0.3060 \\ 0.7651\end{array}\right]$.

Figure 4.4 shows the comparison among a) the polyhedral invariant sets constructed off-line by algorithm 3.1, b) the ellipsoidal invariant sets constructed off-line based on the worst-case performance cost as proposed by Wan and Kothare [6] and c) the ellipsoidal invariant sets constructed off-line based on the nominal performance cost as proposed by Ding et al. [7]. Note that for all algorithms, the invariant sets are constructed by choosing the same sequence of states $x_{i}=\{(0.25,0.25),(0.20,0.20),(0.15,0.15),(0.10,0.10),(0.05,0.05)\}$. It can be observed that the algorithm 3.1 has the largest stabilizable region as compared with other algorithms.

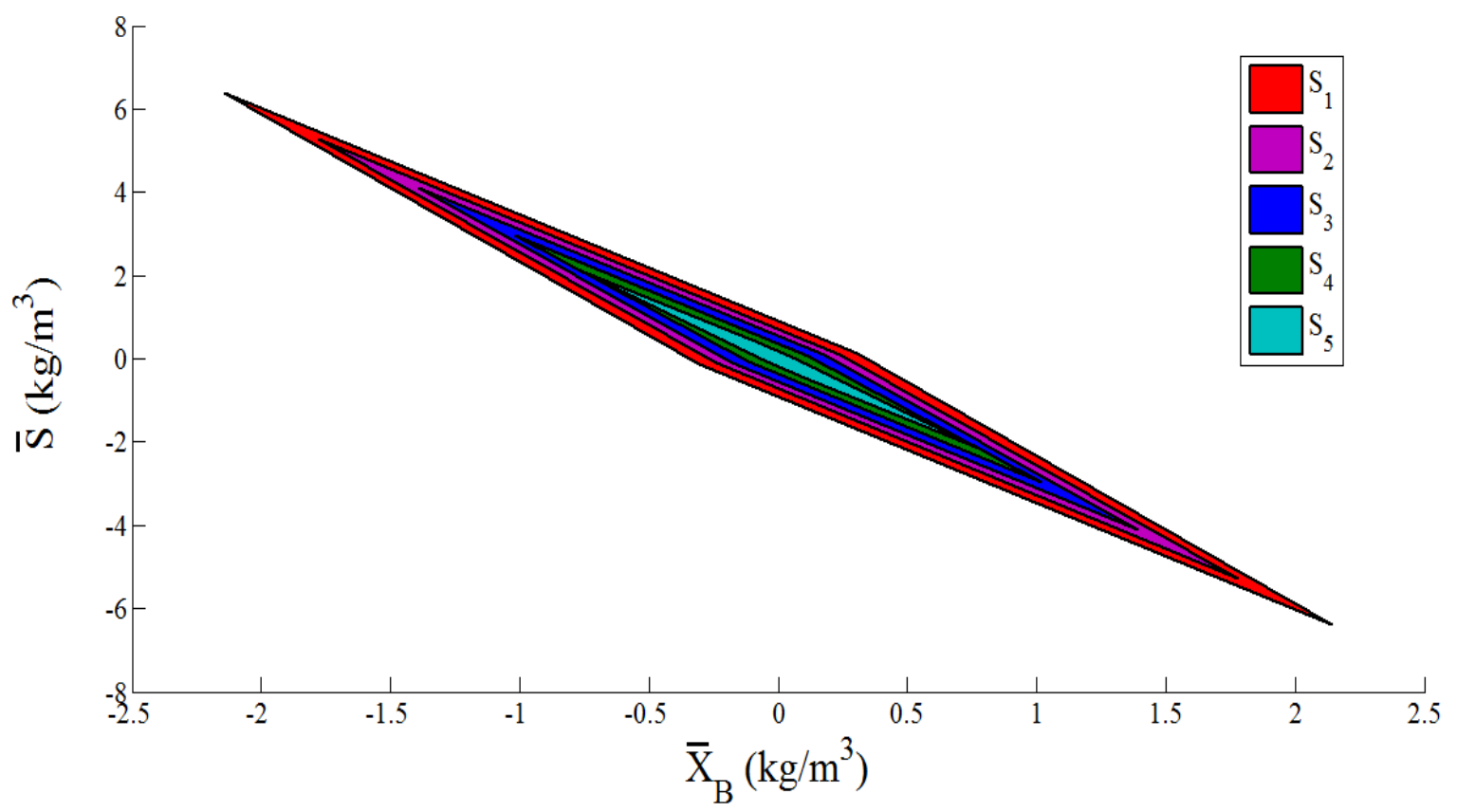

(a) The polyhedral invariant sets $S_{i}, i=1, \ldots, 5$ constructed off-line by algorithm 3.1. 


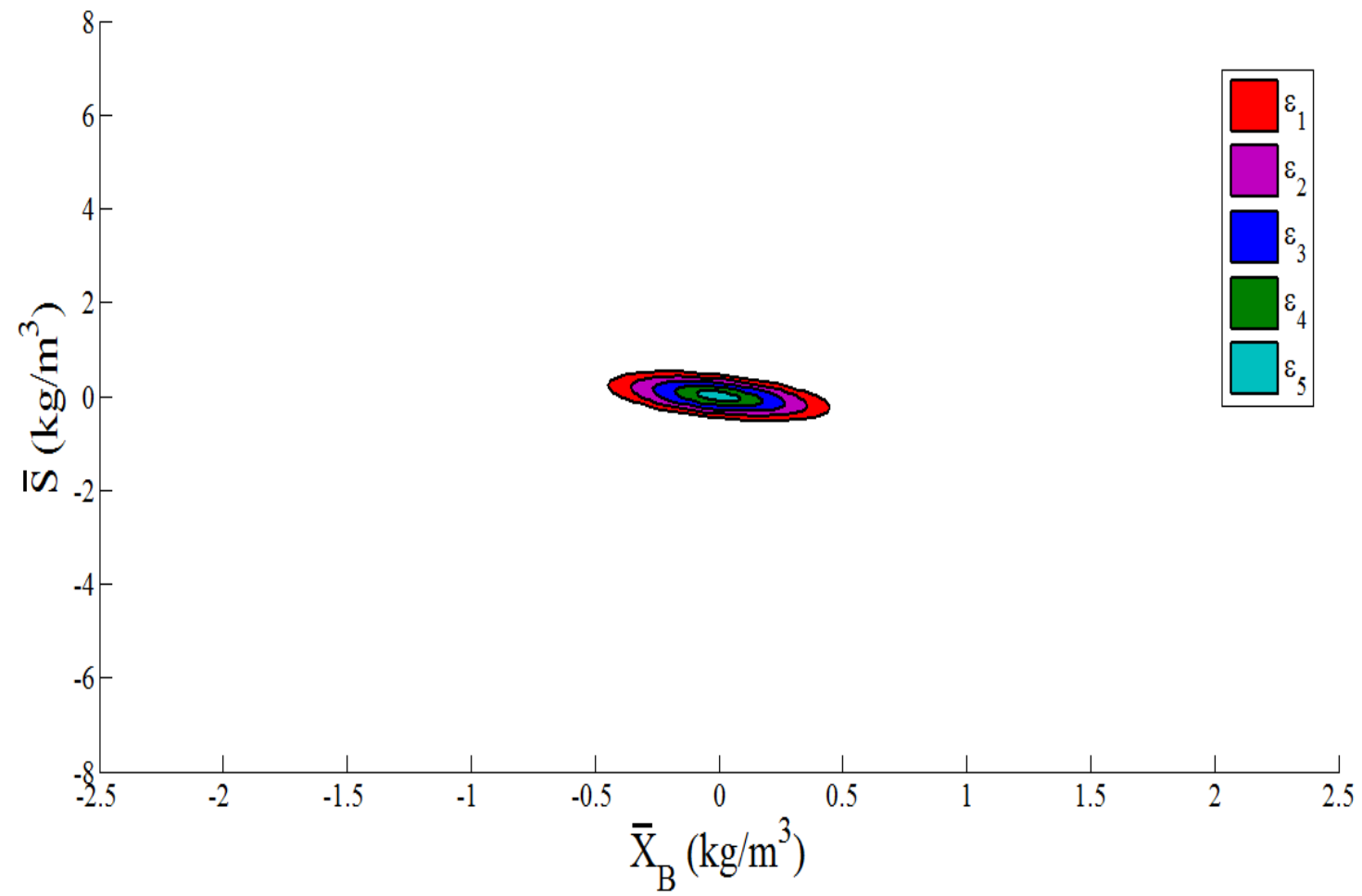

(b) The ellipsoidal invariant sets $\varepsilon_{i}, i=1, \ldots, 5$ constructed off-line based on the worst-case performance cost.

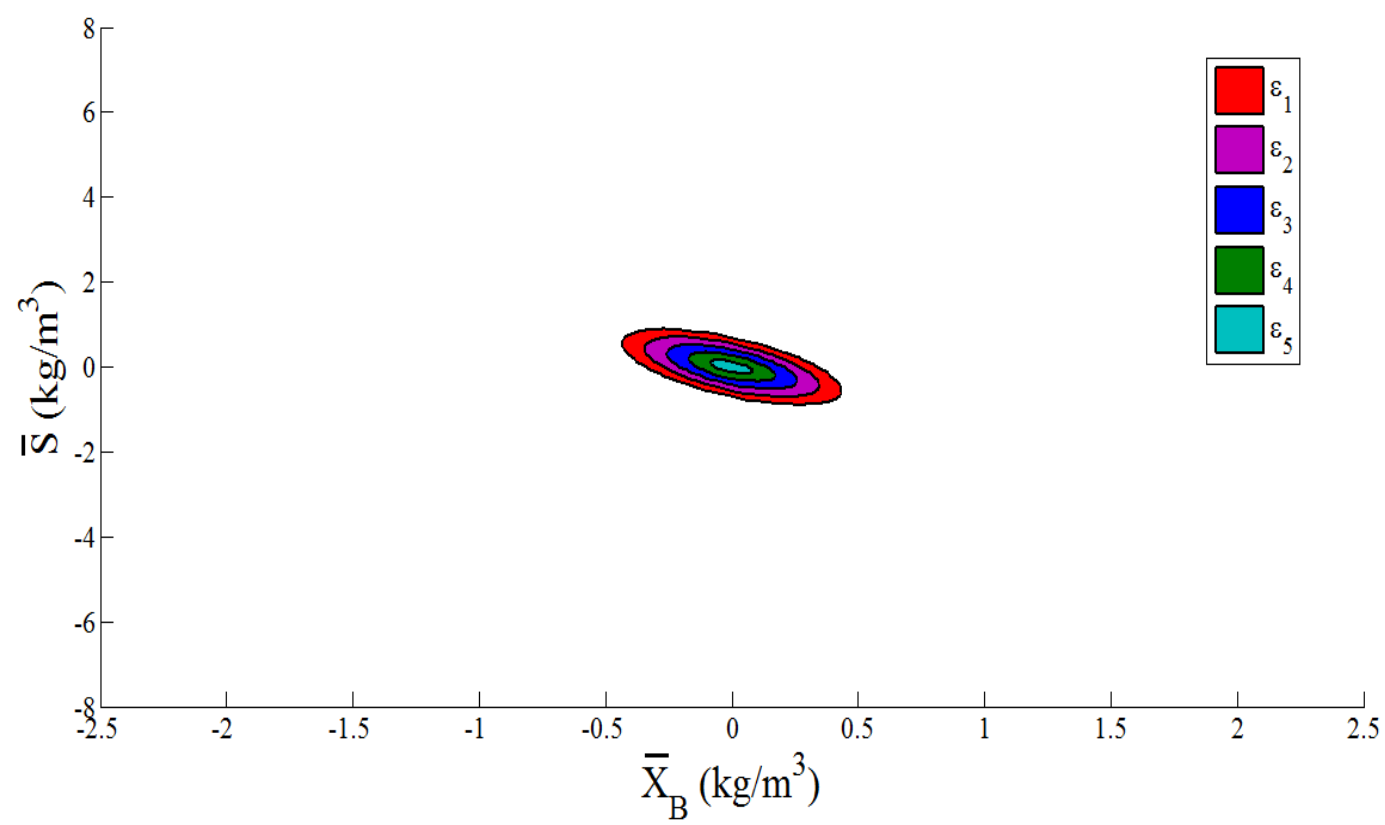

(c) The ellipsoidal invariant sets $\varepsilon_{i}, i=1, \ldots, 5$ constructed off-line based on the nominal performance cost.

Fig. 4.4. The comparison among (a) The polyhedral invariant sets constructed off-line by algorithm 3.1, (b) the ellipsoidal invariant sets constructed off-line based on the worst-case performance cost, and (c) the ellipsoidal invariant sets constructed off-line based on the nominal performance cost. For all algorithms, the invariant sets are constructed by choosing the same sequence of states $x_{i}=\{(0.25,0.25),(0.20,0.20),(0.15,0.15),(0.10,0.10),(0.05,0.05)\}$. 
Figure 4.5 shows the stabilizable regions of feedback gains $K_{1}$ and $K_{2}$. In this example, $K_{2}$ is larger than $K_{1}$ because $K_{2}$ is computed by using the state which is closer to the origin than $K_{1}$. Note that each $K_{\mathrm{i}}$ of algorithm 3.1 and $K_{\mathrm{i}}$ of an ellipsoidal off-line robust MPC algorithm based on the nominal performance cost are identical because they are all derived by minimizing the nominal performance cost. In comparison, each $K_{\mathrm{i}}$ of an ellipsoidal off-line robust MPC algorithm based on the worst-case performance cost is derived by minimizing the worst-case performance cost and hence it can entail a certain degree of conservativeness because the effect of plant uncertainty is overestimated. It can be observed from the figure that an ellipsoidal off-line robust MPC algorithm based on the worst-case performance cost and an ellipsoidal offline robust MPC algorithm based on the nominal performance cost cannot stabilize the state at point A. This is due to the fact that the state at point $\mathrm{A}$ is not contained in the largest invariant ellipsoid $x_{\text {point A }} \notin \varepsilon_{1}$. In comparison, algorithm 3.1 can stabilize the state at point A because the state is contained in the largest polyhedral invariant set $x_{\text {point A }} \in S_{1}$. It can also be observed from the figure that if we start at point $\mathrm{B}$, an ellipsoidal off-line robust MPC algorithm based on the worst-case performance cost and an ellipsoidal off-line robust MPC algorithm based on the nominal performance cost can stabilize the state by using the lowest feedback gain $K_{1}$ because $x_{\text {point B }} \in \varepsilon_{1}$. In comparison, the algorithm 3.1 can stabilize the state by using higher feedback gain $K_{2}$ due to the fact that $x_{\text {point B }} \in S_{2}$. In this circumstance, algorithm 3.1 can adopt higher feedback gain as compared with an ellipsoidal off-line robust MPC algorithm based on the worst-case performance cost and an ellipsoidal off-line robust MPC algorithm based on the nominal performance cost. Although the stabilizable region of algorithm 3.1 is significantly larger than the stabilizable region of an ellipsoidal off-line robust MPC algorithm based on the worst-case performance cost, there are some points in the stabilizable region of an ellipsoidal off-line robust MPC algorithm based on the worst-case performance cost that are not contained in the largest polyhedral invariant set of algorithm 3.1, for example $x_{\text {point } C} \notin S_{1}$. This is due to the fact that the feedback gain of algorithm 3.1 is derived by minimizing the nominal performance cost while the feedback gain of an ellipsoidal off-line robust MPC algorithm based on the worst-case performance cost is derived by minimizing the worst-case performance cost. Thus, it's not necessary for both algorithms to have the overlapping stabilizable region.

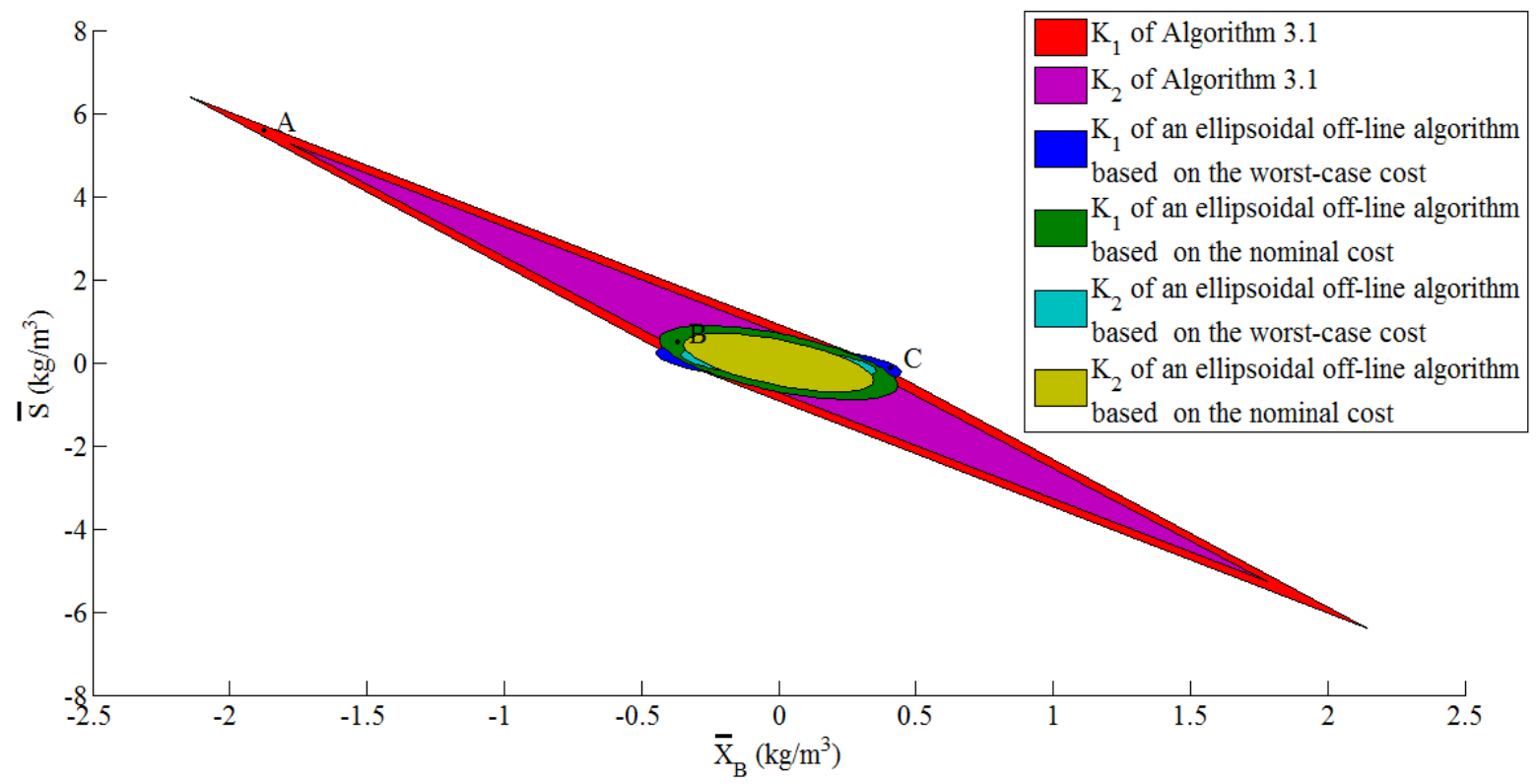

Fig. 4.5. The stabilizable regions of feedback gains $K_{1}$ and $K_{2}$.

Table 4.3 shows the cumulative cost $\sum_{i=0}^{\infty} x(i)^{T} \Theta x(i)+u(i)^{T} R u(i)$ obtained from the simulations when $\mu_{\text {max }}(k)$ is randomly time-varying between $0.01 \leq \mu_{\max }(k) \leq 0.99$. It can be observed that algorithm 3.1 can achieve better control performance as compared with an ellipsoidal off-line robust MPC algorithm based on the worst-case performance cost and an ellipsoidal off-line robust MPC algorithm based on the 
nominal performance cost. This is due to the fact that for each chosen state $x_{i}$, the stabilizable region of polyhedral invariant set constructed off-line by algorithm 3.1 is significantly larger than the stabilizable region of an ellipsoidal invariant set constructed off-line by an ellipsoidal off-line robust MPC algorithm based on the worst-case performance cost and the stabilizable region of an ellipsoidal invariant set constructed off-line by an ellipsoidal off-line robust MPC algorithm based on the nominal performance cost. As previously discussed in example 4.1, algorithm 3.1 can adopt higher feedback gain as compared with an ellipsoidal off-line robust MPC algorithm based on the worst-case performance cost and an ellipsoidal off-line robust MPC algorithm based on the nominal performance cost.

Table 4.3. The cumulative cost $\sum_{i=0}^{\infty} x(i)^{T} \Theta x(i)+u(i)^{T} R u(i)$ in Example 4.2.

\begin{tabular}{lr}
\hline Algorithm & $\begin{array}{r}\text { Cumulative } \\
\text { Cost }\end{array}$ \\
\hline Algorithm 3.1 & 0.093 \\
An ellipsoidal off-line algorithm based on the nominal cost & 0.101 \\
An ellipsoidal off-line algorithm based on the worst-case cost & 0.109 \\
\hline
\end{tabular}

\section{Conclusions}

In this paper, we have presented an off-line synthesis approach to robust constrained model predictive control using polyhedral invariant sets. The on-line computational time is reduced by computing off-line a sequence of state feedback control laws corresponding to a sequence of polyhedral invariant sets. The nominal model of the plant is included in the problem formulation in order to improve control performance. The controller design is illustrated with two examples of the continuous stirred-tank reactor and the bioreactor in fermentation process. The proposed algorithm is compared with the ellipsoidal offline robust model predictive control algorithms. The results show that the proposed algorithm can achieve better control performance. Moreover, a significantly larger stabilizable region is obtained.

\section{Acknowledgment}

This work was supported by Computational Process Engineering Research Group, Special Task Force for Activating Research (STAR), Chulalongkorn University Centenary Academic Development Project.

\section{References}

[1] X. Li and T.E. Marlin, "Model predictive control with robust feasibility," J. Process Contr., vol. 21, pp. 415-435, 2011.

[2] M. V. Kothare, V. Balakrishnan, and M. Morari, "Robust constrained model predictive control using linear matrix inequalities," Automatica, vol. 32, pp. 1361-1379, 1996.

[3] T. A. T. Do and D. Banjerdpongchai, "Robust constrained model predictive control for uncertain linear time-varying systems using multiple lyapunov functions," in Proceedings of SICE-ICASE International Joint Conference, Korea, 2006, pp. 908-913.

[4] A. C. Brooms, B. Kouvaritakis, and Y. I. Lee, "Constrained MPC for uncertain linear systems with ellipsoidal target sets,” Syst. Control Lett., vol. 44, pp. 157-166, 2001.

[5] P. Bumroongsri and S. Kheawhom, "MPC for LPV systems based on parameter-dependent Lyapunov function with perturbation on control input strategy," Engineering Journal, vol. 16, pp. 61-72, 2012.

[6] Z. Wan and M.V. Kothare, "An efficient off-line formulation of robust model predictive control using linear matrix inequalities," Automatica, vol. 39, pp. 837-846, 2003.

[7] B. Ding, Y. Xi, M. T. Cychowski, and T. O. Mahony, "Improving off-line approach to robust MPC based-on nominal performance cost," Automatica, vol. 43, pp. 158-163, 2007.

[8] D. Angeli, A. Casavola, G. Franze, and E. Mosca, "An ellipsoidal off-line MPC scheme for uncertain polytopic discrete-time systems," Automatica, vol. 44, pp. 3113-3119, 2008. 
[9] P. Bumroongsri and S. Kheawhom, "An ellipsoidal off-line model predictive control strategy for linear parameter varying systems with applications in chemical processes," Syst. Control Lett., vol. 61, pp. 435442, 2012.

[10] B. Pluymers, J. A. Rossiter, J. A. K. Suykens, and B. D. Moor, "The efficient computation of polyhedral invariant sets for linear systems with polytopic uncertainty," in Proceedings of the American Control Conference, Portland, 2005, pp. 804-809.

[11] J. F. Sturm, "Using Sedumi 1.02, a MATLAB toolbox for optimization over symmetric cones," Optim. Method Softw., vol. 11, pp. 625-653, 1999.

[12] J. Löfberg, "YALMIP: A toolbox for modelling and optimization in MATLAB," in Proceedings of the 2004 IEEE international symposium on computer aided control systems design, Taipei, Taiwan, 2004, pp. 284289.

[13] M. Galluzzo, B. Cosenza, A. Matharu, and C. Pelayo-Ortiz, "Control of a nonlinear continuous bioreactor with bifurcation by a type-2 fuzzy logic controller," Comput. Chem. Eng., vol. 32, pp. 29862993, 2008. 
